\title{
The hospital frailty risk score is of limited value in intensive care unit patients
}

\author{
Raphael Romano Bruno ${ }^{1}$, Bernhard Wernly ${ }^{2}$, Hans Flaatten ${ }^{3}$, Fabian Schölzel ${ }^{4}$, Malte Kelm ${ }^{1}$ and Christian Jung ${ }^{1 *}$ \\ This comment refers to the article available at https://doi.org/10.1186/s13054-018-2136-4.
}

Keywords: Frailty, Intensive care, Outcome mortality, Outcome non-mortality, Assessment

The identification of patients with frailty is of utmost importance, in particular during intensive care treatment of very old intensive care patients (VOPs). It is quite obvious that tools for this triage process should differ from younger patients. Frailty-not necessarily age-is associated with a negative impact on outcome, especially in critically ill patients [1]. This problem is of great importance as VOPs are one of the fastest growing subgroups in intensive care medicine. We expect an increase in the proportion of the world population older than 60 years from $12 \%$ in 2013 to $21 \%$ in 2050 [2].

Currently, there is an ongoing debate about which tool should be used for this purpose. In this context, we read with great interest about a novel ICD-10code-based algorithm (hospital frailty risk score, HFRS) to identify frail patients at risk [3]. Until now, there has not been any field-testing for its value on the intensive care unit. Therefore, we performed a retrospective analysis and evaluated the impact of HFRS on outcome of ICU patients in our database containing 4381 ICU patients (described previously [4]). We included 1498 patients older than 75 years and calculated HFRS, APACHE-II, and
SAPS-II scores for each patient individually. Survival rates were calculated using uni- and multivariable logistic regression intra-ICU mortality and both uniand multivariable Cox regression analysis to adjust for confounding factors for the long-term combined endpoint of mortality and risk for readmission.

Table 1 demonstrates patients' characteristics. As expected, survivors had significantly lower HFRS than non-survivors. HFRS was significantly associated with adverse outcome (HR 1.09 95\%CI 1.05-1.13; $p<0.001$ ). However, we found no independent association of HFRS after adjustment for APACHE-II scores (HR 1.03 95\%CI $0.98-1.09 p=0.27$ ) or SAPS-II scores (HR $1.0595 \% \mathrm{CI}$ $1.99-1.11 ; p=0.14$ ) in a multivariable model.

This finding contrasts validating studies for the emergency department [5]. Possibly, there is a relevant lack in ICD coding for relevant comorbidities in very old patients on the ICU. In our field testing with realistic conditions in an ICU setting, HFRS does not independently predict risk in ICU patients above 75 years. In conclusion, frailty is complex and its detection crucial, but automatic electronic addition of ICD codes cannot replace the clinical assessment.

\footnotetext{
* Correspondence: Christian.Jung@med.uni-duesseldorf.de

${ }^{1}$ Division of Cardiology, Pulmonology, and Vascular Medicine, Medical

Faculty, University Hospital Düsseldorf, Düsseldorf, Germany

Full list of author information is available at the end of the article
} 
Table 1 Baseline characteristics

\begin{tabular}{lllll}
\hline & Survivors & Non-survivors & Total cohort & $P$ value \\
\hline HFRS & $2.9( \pm 3.3, n=1259)$ & $4.1( \pm 3.5 ; n=239)$ & $3.1( \pm 3.36 ; n=1498)$ & $<0.001$ \\
Sex (male, [\%]) & $60 \%$ & $54 \%$ & $59 \%$ & 0.12 \\
Age $($ mean, [years]) & $80.9( \pm 4.2 ; n=1259)$ & $81.5( \pm 4.33 ; n=239)$ & $81.0( \pm 4.2 ; n=1498)$ \\
Lactate $[\mathrm{mmol} / \mathrm{L}]$ & $2.0( \pm 1.6 ; n=1029)$ & $6.1( \pm 5.41 ; n=184)$ & $2.7( \pm 3.0 ; n=1213)$ & 0.07 \\
Creatinine $[\mathrm{mmol} / \mathrm{L}]$ & $149.4( \pm 118.8 ; n=1195)$ & $206.9( \pm 83.5 ; n=229)$ & $158.6( \pm 122.1 ; n=1424)$ & $13.7( \pm 10.2 ; n=1424)$ \\
Urea $[\mathrm{mmol} / \mathrm{L}]$ & $12.9( \pm 9.8 ; n=1196)$ & $17.7( \pm 11.05 ; n=228)$ & $25.0( \pm 6.5 ; n=551)$ & $<0.001$ \\
Albumin $[\mathrm{g} / \mathrm{L}]$ & $25.9( \pm 6.2 ; n=448)$ & $21.1( \pm 6.27 ; n=103)$ & $14 \%$ & $<0.001$ \\
Use of catecholamine & $13 \%$ & $18 \%$ & $30 \%$ & $<0.001$ \\
Invasive ventilation & $23 \%$ & $59 \%$ & $11 \%$ & $<0.001$ \\
Hemodialysis & $8 \%$ & $23 \%$ & $<0.001$
\end{tabular}

HFRS hospital frailty risk score. Normally distributed data points are expressed as mean \pm standard deviation. Differences between independent groups were calculated using ANOVA. Categorical data are expressed as numbers (percentage)

\section{Acknowledgements}

None.

\section{Authors' contributions}

RRB, BW, and CJ designed the study; acquired, analyzed, and interpreted the data; and wrote and revised the manuscript. MK and HF analyzed and interpreted the data and revised the manuscript. FS supported us technically in analyzing the data and revised the manuscript. All authors read and approved the final manuscript.

\section{Funding}

None.

\section{Availability of data and materials}

The datasets used and/or analyzed during the current study are available from the corresponding author on reasonable request.

\section{Ethics approval and consent to participate}

The local ethics committee of the Jena University Hospital had approved the study (\#2762-02/10).

\section{Consent for publication}

Not applicable.

\section{Competing interests}

The authors declare that they have no competing interests.

\section{Author details}

${ }^{1}$ Division of Cardiology, Pulmonology, and Vascular Medicine, Medical Faculty, University Hospital Düsseldorf, Düsseldorf, Germany. ${ }^{2} \mathrm{Clinic}$ of Internal Medicine II, Department of Cardiology, Paracelsus Medical University of Salzburg, Salzburg, Austria. ${ }^{3}$ Department of Anesthesia and Intensive Care, Haukeland University Hospital, Bergen, Norway. ${ }^{4}$ Division of

Gastroenterology, Hepatology and Infectiology, Medical Faculty, University Hospital Düsseldorf, Düsseldorf, Germany.

Received: 14 May 2019 Accepted: 18 June 2019

Published online: 02 July 2019

\section{References}

1. So RKL, Bannard-Smith J, Subbe CP, Jones DA, van Rosmalen J, Lighthall GK, Investigators MS. The association of clinical frailty with outcomes of patients reviewed by rapid response teams: an international prospective observational cohort study. Crit Care. 2018;22(1):227.

2. Leblanc G, Boumendil A, Guidet B. Ten things to know about critically ill elderly patients. Intensive Care Med. 2017:43(2):217-9.

3. Gilbert T, Neuburger J, Kraindler J, Keeble E, Smith P, Ariti C, Arora S, Street A, Parker S, Roberts HC, et al. Development and validation of a hospital frailty risk score focusing on older people in acute care settings using electronic hospital records: an observational study. Lancet. 2018;391(10132): 1775-82.

4. Masyuk M, Wernly B, Lichtenauer M, Franz M, Kabisch B, Muessig JM, Zimmermann G, Lauten A, Schulze PC, Hoppe UC, et al. Prognostic relevance of serum lactate kinetics in critically ill patients. Intensive Care Med. 2019:45(1):55-61.

5. Eckart A, Hauser SI, Haubitz S, Struja T, Kutz A, Koch D, Neeser O, Meier MA, Mueller B, Schuetz P. Validation of the hospital frailty risk score in a tertiary care hospital in Switzerland: results of a prospective, observational study. BMJ Open. 2019;9(1):e026923.

\section{Publisher's Note}

Springer Nature remains neutral with regard to jurisdictional claims in published maps and institutional affiliations. 